\title{
Visualization and Recording of Structural Changes in Hydrated, Living Muscle Myofilaments using the Gas Environmental Chamber
}

\author{
Haruo Sugi*, Takuya Miyakawa ${ }^{2}$, Masaru Tanokura ${ }^{2}$, Shigeru Chaen ${ }^{3}$, Hiroki Minoda ${ }^{4}$ and Tsuyoshi Akimoto \\ ${ }^{1}$ Department of Physiology, School of Medicine, Teikyo University, Tokyo, Japan \\ ${ }^{2}$ Graduate School of Agricultural and Life Sciences, University of Tokyo, Tokyo, Japan \\ ${ }^{3}$ Department of Integrated Sciences in Physics and Biology, College of Humanities and Sciences, Nihon University, Tokyo, Japan \\ ${ }^{4}$ Department of Applied Physics, Tokyo University of Agriculture and Technology, Tokyo, Japan
}

\begin{abstract}
Although more than 50 years have passed since the monumental discovery of ATP-dependent sliding filament mechanism in muscle contraction, the mechanism of cyclic attachment-detachment cycle between myosin heads extending from myosin filaments and corresponding sites on actin filaments still remains to be a matter for debate and speculation. Using the gas environmental chamber (EC) attached to an electron microscope, we have succeeded in recording images of hydrated myosin filaments, with gold particle position markers attached to individual myosin heads. In the absence of ATP, the position of individual myosin heads does not change appreciably with time, indicating stability of time-averaged myosin head mean position. On ATP application, individual myosin heads move parallel to the filament axis by $\sim 6 \mathrm{~nm}$. At both sides of the filament bare region, across which myosin head polarity is reversed, individual myosin heads move away from, but not towards, the bare region, indicating that the observed myosin head movement corresponds to the recovery stroke, associated with reaction, $M+$ ATP $\rightarrow$ M.ADP.Pi. After exhaustion of applied ATP, individual myosin heads return towards their initial position. Recently, we have further succeeded in recording ATPinduced myosin head power stroke in the presence of actin filaments, and have found that the amplitude of power stroke in the isometric condition is $\sim 3 \mathrm{~nm}$, and increases to $\sim 5 \mathrm{~nm}$ at low ionic strength, in accordance with the physiological experiments that $\mathrm{Ca}^{2+}$-activated force increases $\sim$ two fold at low ionic strength. These findings about the novel features of ATP-induced myosin head movement indicate that the EC is an extremely powerful tool, which enables us to visualize and investigate behavior of individual myosin heads in living, hydrated myosin filaments, retaining their physiological function. Finally, we emphasize that our EC work still remains to be the only attempt to investigate function of hydrated macromolecules.
\end{abstract}

Keywords: Muscle contraction; Myosin head recovery stroke; Myosin head power stroke; Gas environmental chamber; Hydrated myosin filament

\section{Introduction}

\section{The Gas environmental chamber}

The gas environmental chamber (EC,or the hydration chamber) is a device to observe and record physical and chemical reactions in water in the electron microscope [1]. Although the EC has been widely

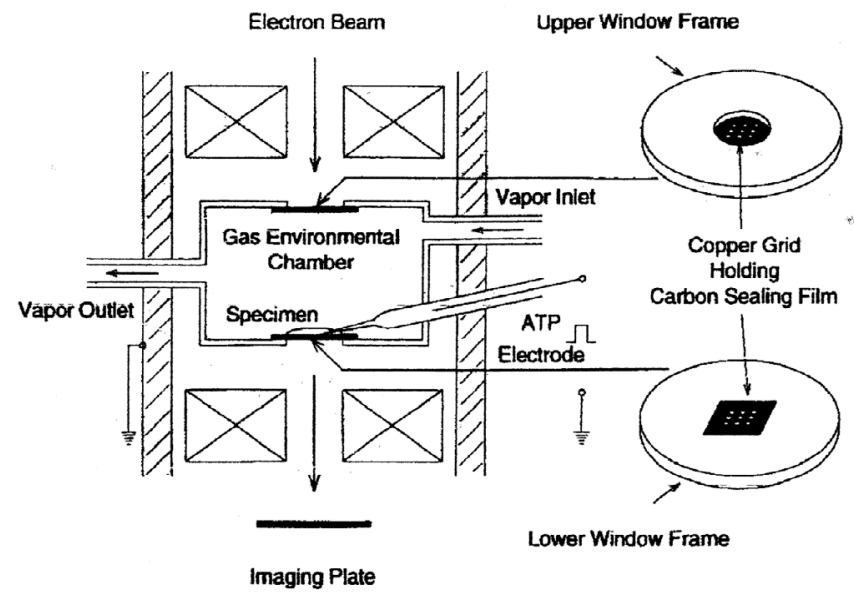

Figure 1: Diagram showing the film-sealed EC. The upper and lower windows (copper grids with nine apertures) are covered with carbon sealing films held on copper grids. The EC contains an ATP-containing glass microelectrode to apply ATP to the specimen iontophoretically. The specimen image is recorded with the imaging plate (IP) [4]. used by materials scientists for in situ recordings of reactions between inorganic substances electron microscopically, its use in medical and biological research fields has not been successful over many years because of a number of technical difficulties. The EC has an enormous potential ability to solve many remaining mysteries in medicine and biology, if high spatial resolution of electron microscope is taken into consideration.

As early as 1980, we started using the EC to study molecular mechanism of muscle contraction with the research group of the late Dr. Akira Fukami, who succeeded in preparing a carbon sealing film suitable for our research work. In this article, we will describe our efforts to establish an EC experimental system to study hydrated muscle proteins, retaining their physiological function, in a chronological order, together with new findings obtained. Although a sealing film made from silicon nitride $\left(\mathrm{Si}_{3} \mathrm{~N}_{4}\right)$ has now become commercially available, and some reports have appeared to observe hydrated biological materials electron microscopically $[2,3]$, we still remains to

*Corresponding author: Haruo Sugi, Department of Physiology, School of Medicine, Teikyo University, Tokyo, Japan, Tel/Fax: +81 48478 4079; E-mail: sugi@kyf.biglobe.ne.jp

Received December 13, 2013; Accepted February 10, 2014; Published February 12, 2014

Citation: Sugi H, Miyakawa T, Tanokura S, Chaen S, Minoda H, et al. (2014) Visualization and Recording of Structural Changes in Hydrated, Living Muscle Myofilaments using the Gas Environmental Chamber. J Nanomed Nanotechnol S5:005. doi:10.4172/2157-7439.S5-005

Copyright: (c) 2014 Sugi $\mathrm{H}$, et al. This is an open-access article distributed under the terms of the Creative Commons Attribution License, which permits unrestricted use, distribution, and reproduction in any medium, provided the original author and source are credited. 


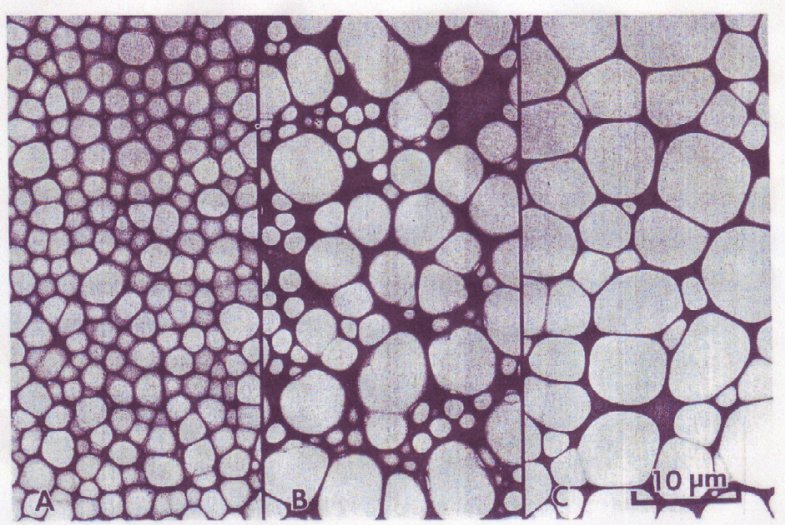

Figure 2: Photomicrographs of the plastic microgrids with holes of smal diameters $(A)$, with holes of non uniform diameters $(B)$, and with holes of fairly uniform diameters $(5-8 \mathrm{~nm})(\mathrm{C})[5]$

A

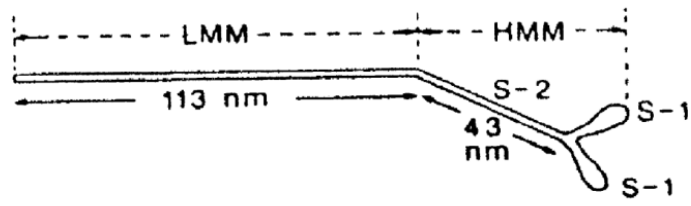

B

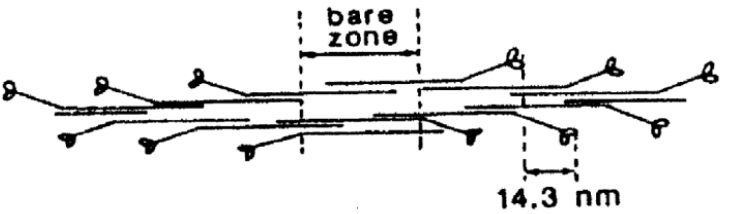

C

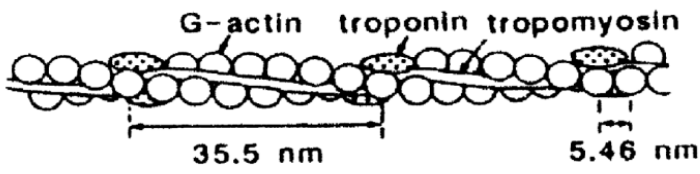

D

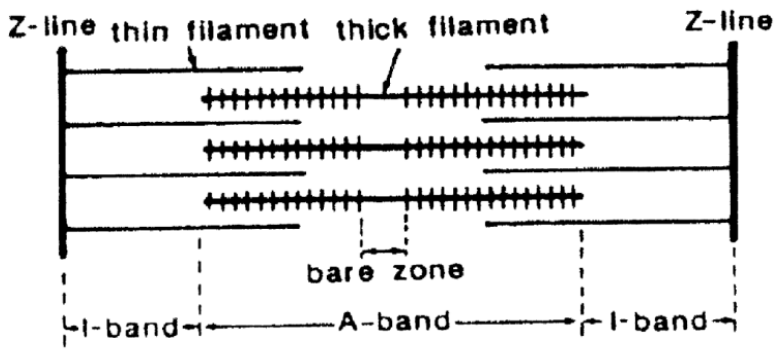

Figure 3: Structure of myosin and actin filaments and their arrangement within a sarcomere. (A) Diagram of a myosin molecule. (B) Arrangement of myosin molecules to form a myosin filament. Note that the myosin filament is symmetrical with respect to central bare zone (or bare region), and that myosin heads (S1) extends from the filament except for bare zone. (C) Arrangement of actin monomers (G-actin) in an actin filament. Note that the actin filament contains tropomyosin and troponin. (D) Arrangement of actin and myosin filaments within a sarcomere. Note that the half sarcomeres at both sides of the bare zone are structural and functional units of striated muscle [10].

be the only research group studying physiological functions of hydrated macromolecules successfully.
Figure 1 is a diagram showing the structure of the EC designed by us [4]. The EC consists of a metal compartment (diameter, $3.5 \mathrm{~mm}$; depth, $0.8 \mathrm{~mm}$ ) with upper and lower window frames (copper grids) to pass electron beam. Each window frame has nine apertures (diameter, $0.1 \mathrm{~mm}$ ), and covered with carbon sealing film, so that the interior of the EC is insulated from high vacuum of electron microscope. The biological specimen is placed on the surface of lower sealing film, and covered with a layer of experimental solution. Water vapor is constantly circulated at a rate of 0.1-0.2 $\mathrm{l} / \mathrm{min}$ through the EC, so that thin layer of experimental solution is in equilibrium with vapor pressure in the ECT to obtain clear image of the specimen, internal pressure of the EC is made 60 -80Torr $[5,6]$. The EC contains a grass capillary microelectrode filled with ATP solution to apply ATP to the specimen. The EC is attached to a $200 \mathrm{kV}$ transmission electron microscope (JEM 2000EX, JEOL).

\section{Carbon sealing film}

In principle, both spatial resolution and contrast of electron micrographs taken by the EC increases with decreasing thickness of carbon sealing film. Preliminary experiments made in Fukami's laboratory indicated that, to obtain a spatial resolution $<1 \mathrm{~nm}$, sealing film thickness should be $15-20 \mathrm{~nm}$. Meanwhile, resistivity of a sealing film against pressure difference decreases sharply with increasing its area. Fukami and Adachi [7] solved this technical problem by using plastic microgrids made from high-molecular organic compound (cellulose acetobutylate), supporting carbon sealing film. Electron micrographs of the microgrids are shown in Figure 2. Microgrids with small (A) or non-uniform holes (B) are unsuitable, while microgrids with fairly uniform holes (diameter, 5-8 nm) (C) are suitable for electron microscopic observation of the specimen placed in the EC. Details of the methods to prepare carbon sealing film by covering the microgrid with a thin layer of carbon sealing film (thickness, $20 \mathrm{~nm}$ ) have been described elsewhere [5,8].

As will be described in this article, we have been using the EC system combined with the carbon sealing film, with a number of novel features about the ATP-induced movement in individual myosin heads, which cannot be obtained by any other experimental methods.

\section{Historical background of research work with the EC}

Muscle structure and the sliding filament mechanism of muscle contraction: In the middle 1950s, H.E. Huxley and Hanson [9] made a monumental discovery that skeletal muscles are composed of hexagonal lattice of actin and myosin filaments, and that muscle contraction results from relative sliding between the two filaments. Considerable progress has been made with respect to structure and function of actin and myosin filaments [10]. As illustrated in Figure $3 \mathrm{~A}$, a myosin molecule is divided into two parts; (1) a long rod called light meromyosin (LMM) and (2) the rest of myosin molecule called heavy meromyosin (HMM), consisting of a short rod (sub-fragment 2 or S2) and two heads (sub-fragment 1 or S1). In a myosin filament, LMM aggregates to form filament backbone, which is polarized in opposite directions on either side of the central part, while the two $S 1$ heads extend laterally from the filament backbone with an interval of $14.3 \mathrm{~nm}$. The central part of myosin filament is called bare region (or bare zone) where the projection of myosin heads is absent (Figure 3B). Meanwhile, an actin filament consists of two helical strands of globular actin monomers (G-actin), which are wound around each other with a pitch of $35.5 \mathrm{~nm}$. The axial separation of actin monomers is $5.46 \mathrm{~nm}$ (Figure 3C). In vertebrate skeletal muscle, actin filaments also contain tropomyosin and troponin. 

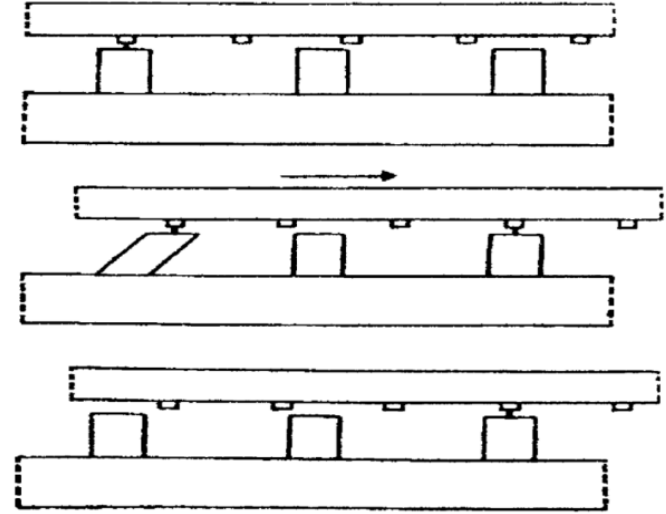

Figure 4: Diagram showing hypothetical attachment-detachment cycle between the myosin heads extending from myosin filaments and the corresponding myosin-binding sites on actin filaments. The periodicity of myosin heads differs from that of myosin biding sites on actin filaments, so that the attachment-detachment cycle takes place asynchronously. The myosin head first attaches to actin filament (top diagram), changes its configuration to move actin filament to he right (middle diagram), and then detaches from actin filament (bottom diagram) [11].
A

D
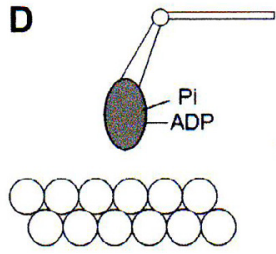

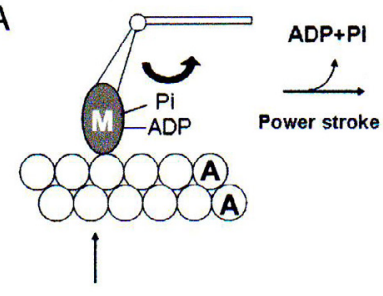

B

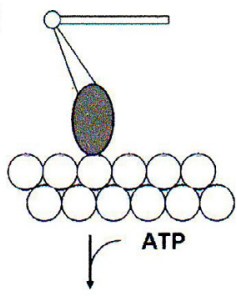

C

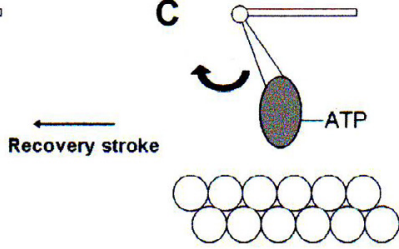

Figure 5: Diagram of the attachment-detachment cycle between myosin heads (M) and actin filaments (A), constructed based on biochemical studies on actomyosin ATPase reactions [16]. Note that power stroke, associated with reaction $\mathrm{AM} \cdot \mathrm{ADP} \cdot \mathrm{Pi} \rightarrow \mathrm{AM}+\mathrm{ADP}+\mathrm{Pi}$, is produced by myosin heads attached to actin filaments (Figure 5A-B), while myosin heads are detached from actin filaments during recovery stroke, associated with reaction $M+A T P \rightarrow$ M.ADP.Pi, takes place [24]

\section{Essential role of myosin heads in muscle contraction}

As shown in Figure 3D, actin filaments extend from Z-line to penetrate in between myosin filaments, located centrally in each sarcomere, i.e. the region between two adjacent Z-lines. Within each sarcomere, the region containing only actin filaments is called the I-band, whereas the region containing myosin filaments and part of actin filaments is called the A-band. It has been shown by a number of investigators that the filament lengths remain constant irrespective of whether a muscle shortens or is stretched. Consequently, the central problem in understanding molecular mechanism of muscle contraction is, what makes actin and myosin filaments slide past each other? Since both actin binding site and ATPase activity are located in two myosin (S1) heads, it is generally believed that myosin heads, extending from myosin filaments towards actin filaments, play a major role in converting chemical energy derived from ATP hydrolysis into mechanical work producing force and motion in muscle.

Figure 4 illustrates hypothetical attachment-detachment cycle between myosin heads extending from myosin filaments and corresponding sites in actin filaments [11]. The attachment-detachment cycle takes place asynchronously, because of the difference in periodicity between myosin heads in myosin filaments and corresponding sites in actin filaments. In this figure, left myosin head is shown to first attach to actin filament (top diagram), changes its configuration in such a way that it moves actin filament to the right (middle diagram), and then detach from actin filament (bottom diagram). Extensive studies have been made to prove the conformational changes of myosin heads coupled with ATP hydrolysis during muscle contraction. Although the methods used include muscle mechanics, time-resolved X-ray diffraction, chemical probes attached to myosin heads, electron microscopy of quickly frozen muscle fibers, crystallographic analysis of nucleotide-dependent structural changes in myosin head crystals, and nucleotide-dependent binding of myosin heads to actin filaments, no clear conclusion has been reached concerning the myosin head conformational changes actually taking place in contracting muscle [12-15].

\section{Shortcomings of biochemical studies on actomyosin ATPase reaction}

On the other hand, a number of biochemical studies have been made concerning reaction steps of ATP hydrolysis of actomyosin (mixture of actin and myosin) in water solution. A most probable sequence of reaction taking place in muscle is diagrammatically illustrated in Figure 5 [16,17], where $A$ and $M$ represent myosin head and actin filament, respectively. $\mathrm{M}$ is connected to myosin filament backbone via myosin subfragment 2 (S2) containing the hinge region, so that $\mathrm{M}$ can rotate around this region (Figure $3 \mathrm{~A}$ and $\mathrm{B}$ ). In relaxed muscle, myosin heads are in the state of M.ADP.Pi, having a long average lifetime $>10 \mathrm{~s}$ due to its slow Pi release [17]; attachment of $\mathrm{M}$ to $\mathrm{A}$ is inhibited by tropomyosin (Figure 5D). When a muscle is activated with $\mathrm{Ca}^{2+}$ to remove tropomyosin inhibition, M.ADP.Pi attaches to A (from $\mathrm{D}$ to $\mathrm{A}$ ), and performs a power stroke (corresponding to conformational changes in Figure 4), associated with reaction AM.ADP.Pi $\rightarrow \mathrm{AM}+\mathrm{ADP}+\mathrm{Pi}$ (from $\mathrm{A}$ to $\mathrm{B}$ ). At the end of power stroke, $\mathrm{M}$ remains attached to $\mathrm{A}$ to form rigor linkage AM. M detaches from A when next ATP comes to bind with $M$ to form M.ATP (from B to C). Then M performs a recovery stroke, associated with reaction $\mathrm{M} \cdot \mathrm{ATP} \rightarrow \mathrm{M} \cdot \mathrm{ADP} \cdot \mathrm{Pi}$ (from $\mathrm{C}$ to $\mathrm{D}$ ), and again attaches to $\mathrm{A}$ (from $\mathrm{D}$ to $\mathrm{A}$ ). The reaction cycle described above includes attachment of $\mathrm{M}$ to and its detachment from, A, and is consistent with the H.E. Huxley's hypothesis shown in Figure 4 [11], and has been generally believed to be applicable to biochemical reactions actually taking place in contracting muscle.

It should, however, be noted that experimental condition in biochemical studies differs too far from that in muscle fibers, because (1) in biochemical experiments, hexagonal myofilament-lattice structure, essential for chemo-mechanical energy conversion, is completely destroyed, (2) actin and myosin concentrations are much higher in muscle than in solutions, and (3) no theoretical treatment has been developed to deal with kinetics of actomyosin ATPase reactions in muscle. To eliminate the gap between muscle biochemistry and physiology, various types of in vitro motility assay systems have been developed, in which isolated HMM or S1 head is made to interact with single actin filaments [18]. Up to the present time, however, the results obtained with these studies have a number of uncertainties about the assumptions to analyze so-called single molecule events and 


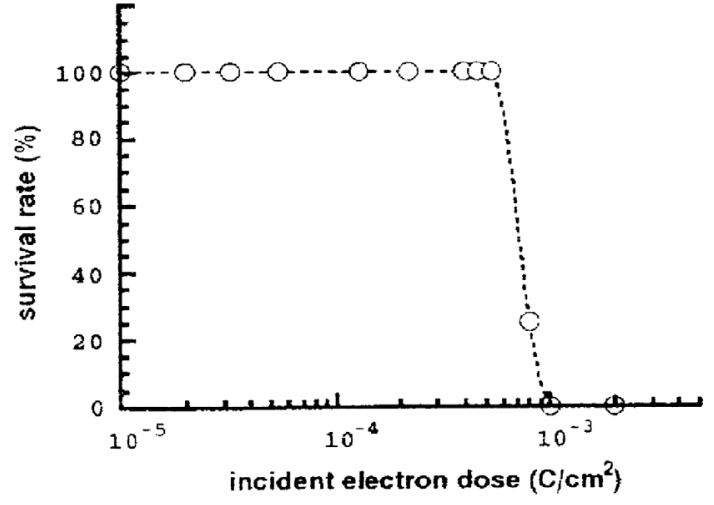

Figure 6: Relation between the total incident electron dose and the surviva rate of muscle myofibrils, expressed as percentage of myofibrils, which contract in response to applied ATP, in the electron microscopic field [22] Note that myofibrils no longer contract in response to ATP, if the electron dose exceeds $5 \times 10^{-4} \mathrm{C} / \mathrm{cm}^{2}$

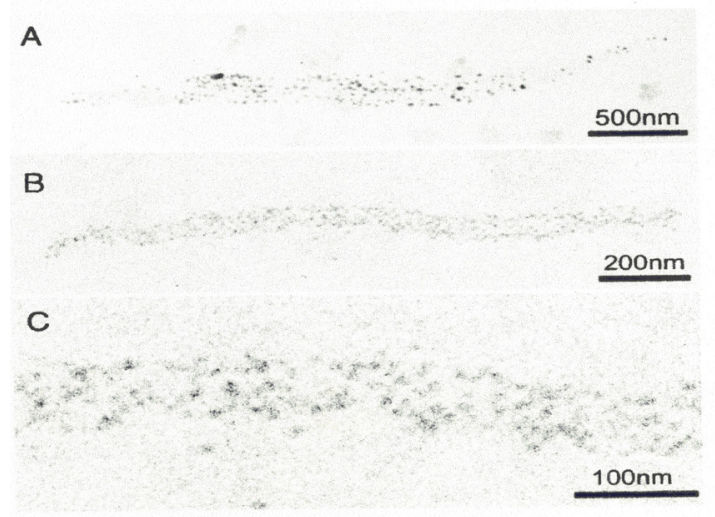

Figure 7: (A and B) Examples of IP records of single bipolar myosin filaments with a number of gold particles attached to individual myosin heads. (C) Enlarged view showing part of myosin filament shown in (B) [24].

the orientation of myosin heads relative to actin filaments. All in all, the performance of myosin heads in producing muscle contraction still remains to be a matter for debate and speculation.

Porter and rower myosins: shortcomings of experimental methods developed for studying processive movement of single porter myosin molecules

In addition to skeletal muscle myosins, a variety of different myosins (unconventional myosin family) exist in the cytoplasm in the form of single molecules to transport substances along cytoplasmic actin filaments. Such myosin molecules move along actin filament in one direction with substances attached to them. The performance of unconventional myosin molecule is compared to a person walking along a track with a load on his back [19]. Such myosins are therefore also called porter (or processive) myosins, while skeletal muscle myosins are called rower myosins, since their heads repeat back-andforth movements asynchronously, just like a group of persons rowing a boat by their asynchronous rowing motion. The processive walking motion of porter myosin molecules is suitable for in vitro motility assay experiments because of their slow velocity of movement along actin filaments. Using appropriate probes, the hand-over-hand processive movement of porter myosin molecules has been recorded under a light microscope $[19,20]$, and also recorded directly under an atomic force microscope [21]. Despite the success in recording the processive movement of porter myosin molecules, however, the techniques used cannot be applied to rower myosins, mainly because rower myosin molecules repeat attachment to, and detachment from, actin filaments asynchronously, and it is impossible to let single skeletal muscle myosin molecules move along actin filaments.

The advantage of using the EC is that the performance of individual myosin heads, located at various regions within a living, hydrated myosin filaments, can be visualized and recorded electron microscopically. While porter myosins work in the form of single molecules, rower myosin molecules in muscle work in concert within myofilament-lattice structures. It may follow from this that the function of rower myosin molecules, i.e. the mechanism of muscle contraction, can best be studied using preparations in which myofilament structures are well preserved. In this connection, the EC as a powerful tool to investigate mechanisms of muscle contraction will be demonstrated in later sections in this article.

\section{Critical electron dose to impair function of muscle proteins}

Although biological specimens placed in the EC can be kept in living, hydrated state, their function is gradually impaired by electron beam irradiation. Before starting the experiments with the EC, it was necessary to determine the critical electron dose to impair function of contractile proteins constituting actin and myosin filaments. This was done in Fukami's group [22]. They observed muscle myofibrils consisting of hexagonal lattice of actin and myosin filaments, in the EC (magnification, 2500X), and activated them to contract with ATP, at various times after beginning of electron beam irradiation. The results are summarized in Figure 6. When the total incident electron dose is $<5 \times 10^{-4} \mathrm{C} / \mathrm{cm}^{2}$, all the myofibrils in the electron microscopic field contract in response to applied ATP. If, however, the total incident electron dose is further increased, the ATP-induced myofibrillar contraction disappears in a nearly all-or-none manner, while the myofibrils show no appreciable change in appearance. The same critical electron dose to impair physiological function of myofibrils has been confirmed by us with respect to both the ATP-induced myosin head movement and the ATPase activity in hydrated myosin filaments placed in the EC [4].

Based on the results shown in Figure 6, electron microscopic observation and recording of the specimen placed in the EC is made with a total incident electron dose $<5 \times 10^{-4} \mathrm{C} / \mathrm{cm}^{2}$, being well below the critical dose to impair function of actin and myosin filaments. To fulfill this condition, the specimen in the EC should be observed with extremely weak electron beam intensities (at the fluorescent screen) $<5 \times 10^{-4} \mathrm{C} / \mathrm{cm}^{2}$. As procedures to find and focus specimens in the electron microscopic field are time-consuming, we have to limit electron microscopic magnification to $10,000 x$.

\section{Experimental methods to record ATP-induced movement of individual myosin heads in hydrated myosin filaments}

Recording of myosin filaments with myosin heads positionmarked with site-directed antibodies and gold particles: Hydrated specimens in the EC should be observed under unstained conditions. It is therefore essential to position-mark myosin heads in hydrated myosin filaments by some means. Fortunately, we can use site-directed antibodies (IgG), prepared by Sutoh et al. [23] for electron microscopic 
A

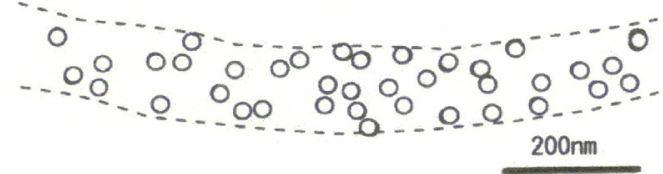

B

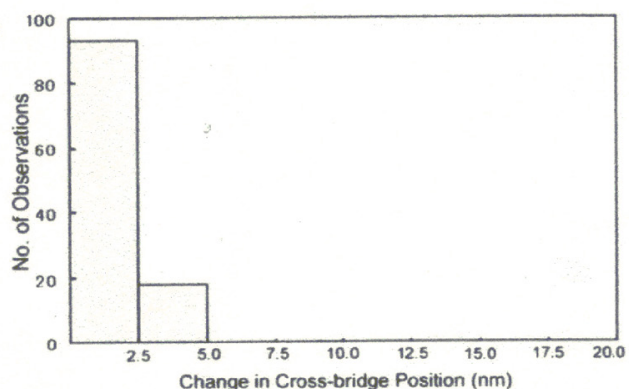

Figure 8: Stability of time-averaged position in individual myosin heads in the absence of ATP. (A) comparison of myosin head positions between the two IP records of the same filament. Open and filled circles (diameter, 20nm) are drawn around the center of mass position of each gold particle, serving to position-mark each myosin head, in the first and the second IP records, respectively. In this and subsequent figures, broken lines indicate contour of myosin filament. Note that filled circles are barely visible due to almost complete overlap of open circles over filled circles. (B) Histogram showing distribution of distances between the center of mass positions of particles in the first and the second IP records [24].

mapping of myosin head. Colloidal gold particles (EY Laboratories, diameter, 15 or $20 \mathrm{~nm}$, coated with protein A serving as a paste to connect different substances) are effectively attached to various regions in individual myosin heads via the antibodies. Since native myosin filaments are too thin (diameter, $15 \mathrm{~nm}$ ) to record with a magnification of $10,000 \mathrm{x}$, and have a tendency to curl and aggregate, we use synthetic myosin filaments prepared by polymerizing rabbit skeletal muscle myosin molecules at low ionic strength solutions (diameter, 50-200 $\mathrm{nm})$ [24]. The images of filaments, with a number of gold particles on them, are recorded with the imaging plate (IP, PIX system, JEOL) with an exposure time of $0.1 \mathrm{~s}$ with a beam intensity of $1-2 \times 10^{-12} \mathrm{~A} / \mathrm{cm}^{2}$. At a magnification of $10,000 \mathrm{x}$, the pixel size of imaging plate is $2.5 \times 2.5 \mathrm{~nm}$. Due to limitation of critical electron dose, the recording of the same filaments can only be made up to 4 times. Temperature of ATP-free experimental solution covering the filaments is kept at $26-28^{\circ} \mathrm{C}[24]$.

\section{Data analysis}

The average number of electrons reaching each pixel during the exposure time is $\sim 10$. Reflecting this electron statistics, each gold particle image consists of $20-50$ pixels. The center of mass position for each particle is determined as the coordinates (two significant figures; accuracy, $\sim 0.6 \mathrm{~nm}$ ) within a single pixel where the center of mass position is located, and these coordinates representing the particle position are taken as the position of myosin head. The change in position of myosin head is compared between the two IP records of the same filaments $[4,24]$.

\section{Application of ATP}

ATP is applied to the filaments iontophonetically by passing a current pulse (intensity, $10 \mathrm{nA}$; duration, 1s) to a glass capillary microelectrode filled with $100 \mathrm{mM}$ ATP, so that ATP ions carrying the current pulse go out of the electrode, and reach the filaments by diffusion in the experimental solution. The total amount of ATP released from the electrode and the ATP concentration around the filaments (at 30 s after the onset of current pulse) is estimated to be $\sim 10^{-14} \mathrm{~mol}$ and $<5$ $\mu \mathrm{M}$, respectively. The methods described above are developed during the course of our preliminary experiments on the ATP-induced myosin head movement in myosin-paramyosin core complex filaments [4]. The results of this work are not mentioned in this article, because the results obtained from complex filaments bear no direct relation to skeletal muscle contraction.

\section{Novel features of ATP-induced movement in individual myosin heads as revealed by experiments using the EC}

Stability of myosin head mean position in hydrated synthetic myosin filaments: Figure 7 is a typical IP record of single synthetic myosin filament placed in the EC. The filaments (diameter, 50$200 \mathrm{~nm}$ at the middle; length, 0.1-3.5 $\mu \mathrm{m}$ ) are spindle-shaped with tapered ends, and a number of gold particles, serving as myosin head position- markers can be seen to distribute along the fiber length. In this particular case, myosin heads are position-marked with antibody attaching to junctional peptide between 50 - and $20-\mathrm{kDa}$ segments of myosin heavy chain, located near the distal end of myosin head [24]. The image of each gold particle consists of 20-50 dark pixels with a wide range of gradation.

By comparing two IP records of the same filament taken at an interval of 5-10 min, the center of mass position of each particle (and therefore each myosin head) has been shown not to change appreciably with time. Figure 8A shows profile of a part of myosin filament, on which a circle of $20 \mathrm{~nm}$ diameter is drawn around the center of mass position of each gold particle obtained from the two IP records. It can be seen that the circles representing the center of mass position of the same particle overlap with each other almost completely, indicating
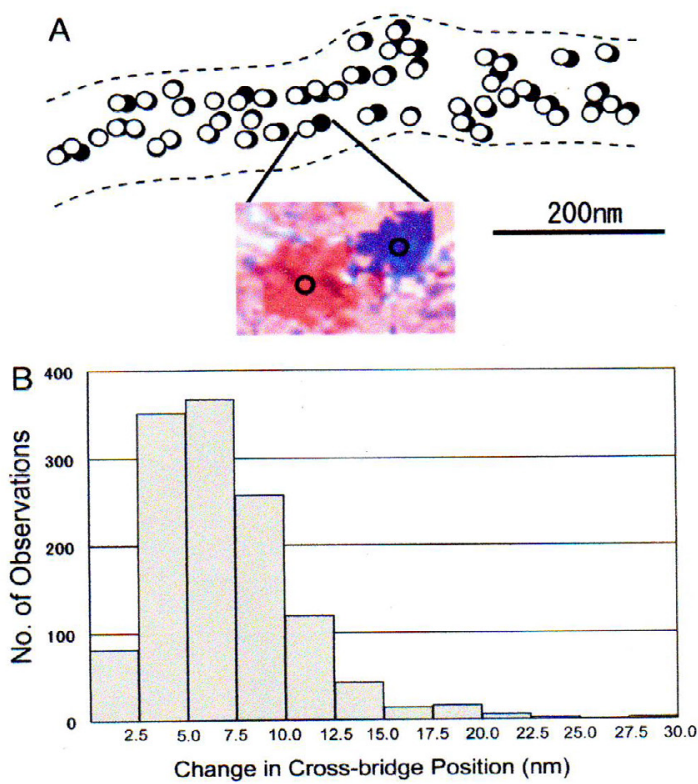

Figure 9: ATP-induced myosin head movement. (A) Comparison of the myosin head position between the two IP records, taken before and after application of ATP. Open and filled circles (diameter, $20 \mathrm{~nm}$ ) are drawn around the center of mass positions of the same particle before and after ATP application, respectively. Inset is an example of superimposed IP records showing the change in position of the same gold particle, colored red (before ATP application) and blue (after ATP application) [24]. (B) Histogram showing the amplitude distribution of ATP-induced myosin head movement. 


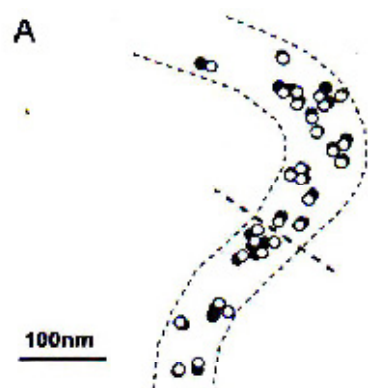

B

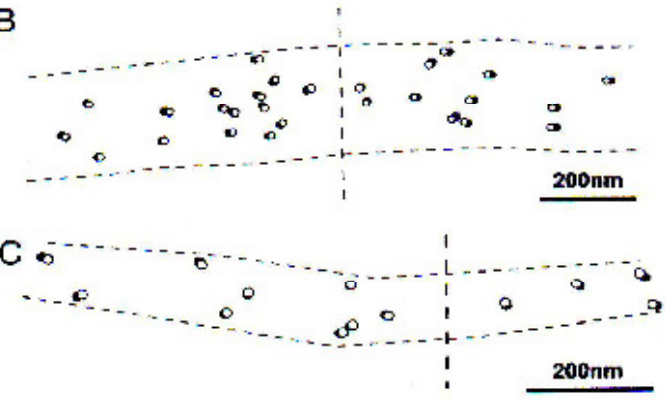

Figure 10: Examples of IP records showing the reversal in the direction of ATP-induced myosin head movement across the filament bare region. Open and filled circles (diameter, 20nm) are drawn around the center of mass positions of the same particles before and after ATP-application, respectively. Note that myosin heads move away from, but not toward, the filament bare region (indicated by broken lines) [24].

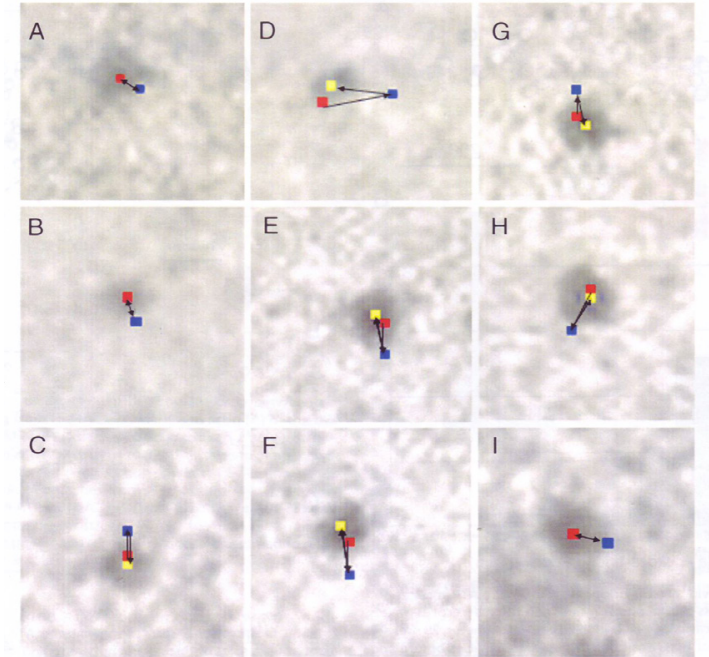

Figure 11: Records showing sequential changes in position of 9 different pixels (each $2.5 \times 2.5 \mathrm{~nm}$ ) where the center of mass positions of corresponding different particles are located. In each frame, the pixel positions are recorded 3 times, i.e. before ATP application, during ATP application, and after exhaustion of applied ATP. Changes in position of pixels in the first (red), second (blue), and third (yellow) records indicate changes in myosin head position before ATP application, during ATP application, and after exhaustion of applied ATP. Directions of each myosin head movement is indicated by arrow. Note that the myosin heads return toward their initial position after exhaustion of ATP [24].

that the center of mass position of each myosin head remains almost unchanged with time. Figure $8 \mathrm{~B}$ is a histogram showing distribution of change in the center of mass position of gold particles (i.e. myosin heads) between the two IP records of the same filaments. Change in position of individual myosin heads are shown to be $<2.5 \mathrm{~nm}$ in the majority of myosin heads examined [24].

These results indicate that (1) myosin filaments are firmly fixed on the carbon sealing film, and (2) despite thermal fluctuation, timeaveraged mean position in individual myosin head remains almost unchanged. The presence of definite equilibrium position not only provides favorable condition for each myosin head to generate force and motion in muscle, but also gives us favorable condition to determine the amplitude of ATP-induced myosin head movement unambiguously.

ATP-Induced movement in individual myosin heads in hydrated synthetic myosin filaments amplitude of ATP-induced myosin head movement: Based on the stability of myosin head mean position, myosin head movement associated with ATP hydrolysis, can be visualized and recorded by measuring the change in position of individual myosin heads in response to iontophonetically applied ATP. This can be done by comparing two IP records of the same filaments, one taken before while the other taken at 40-60s after the onset of current pulse to ATP electrode. A typical result is shown in Figure 9A, in which open and filled circles were drawn around the center of mass positions of the same particles before and after ATP application, respectively. In most cases, individual myosin heads moved nearly in parallel with the filament axis in one direction. As shown in Figure 9B, the amplitude distribution of ATP-induced myosin head movement exhibits a peak at 5-7.5 $\mathrm{nm}$. The average amplitude of ATP-induced myosin head movement (excluding values $<2.5 \mathrm{~nm})$ is $6.5 \pm 3.7 \mathrm{~nm}$ (mean $\pm \mathrm{SD}, \mathrm{n}=1210$ ) [24]. In these experiments, particles located at both upper and lower sides of the filaments are equally in focus in the electron microscopic field. Myosin heads located on the upper surface of the filaments may move almost freely in response to ATP, while those located on the lower surface may attach to carbon sealing film and their movement would be considerably reduced in amplitude. If this explanation is correct, the amplitude of freely moving myosin heads in response to ATP would be well above $7.5 \mathrm{~nm}$.

Reversal in direction of ATP-induced myosin head movement across the filament bare region :We have also succeeded in recording ATP-induced myosin head movement at both sides of the bare region located at the center of myosin filaments. Examples of the results are shown in Figure 10. It will be seen that, in response to ATP, individual myosin heads are observed to move away from, but not toward, the bare region. As the experiments are performed in the absence of actin filaments, the observed myosin head movement away from the bare region corresponds to myosin head recovery stroke, associated with reaction $M+A T P \rightarrow M \cdot A D P \cdot P i$, i.e. reaction from $B$ to $D$ in Figure 5). This result is therefore regarded to be the first direct electron microscopic demonstration of myosin head recovery stroke [24]. We emphasize that such direct demonstration of myosin head recovery stroke has not been possible by any other experimental methods.

Reversibility of ATP-induced myosin head movement: ATPinduced myosin head movement has been shown to be reversible. The reversibility is ascertained by taking three IP records of the same myosin filaments in the following sequence: (1) before ATP application; (2) at 40-60s after the onset of current pulse to the ATP-containing electrode, i.e. during ATP application; and (3) at 5-6 min after ATP application, i.e. after complete exhaustion of applied ATP. In this experiment, hexokinase (50 units/ml) and D-glucose $(2 \mathrm{mM})$ were added to experimental solution to eliminate contamination of ATP [24].

Examples of sequential changes in position of different pixels $(2.5 \times 2.5 \mathrm{~nm}$ each $)$, where the center of mass positions of 


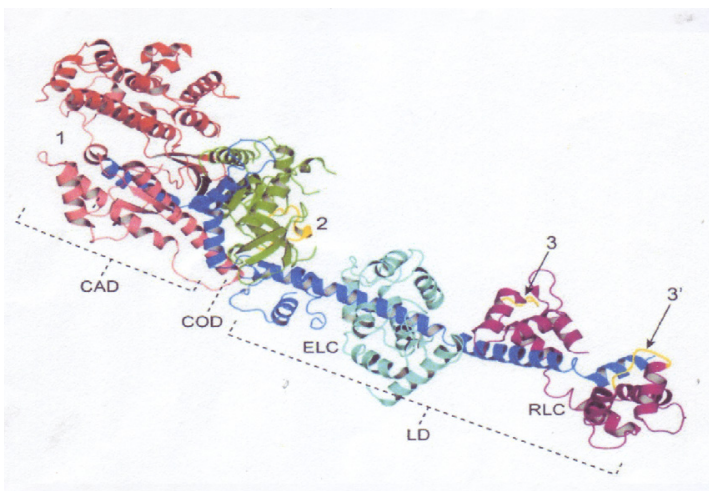

Figure 12: Myosin head structure showing approximate regions of attachment of antibodies 1, 2 and 3, indicated by numbers 1, 2 and 3 and 3', respectively. The catalytic domain (CAD) consists of 25k-Da (green), 50-kDa (red), and part of $20-\mathrm{kDa}$ (dark blue) fragments of myosin heavy chain, while the lever arm domain (LD) consists of the rest of 20-kDa fragment and essential (ELC, light blue) and regulatory (RLC, magenta) light chains. CAD and LD are connected via converter domain (COD). Location of peptides around Lys 83 in COD and that of two peptides (Met58 Ala70 and Leu106 Phe120) in LD are colored yellow [26].

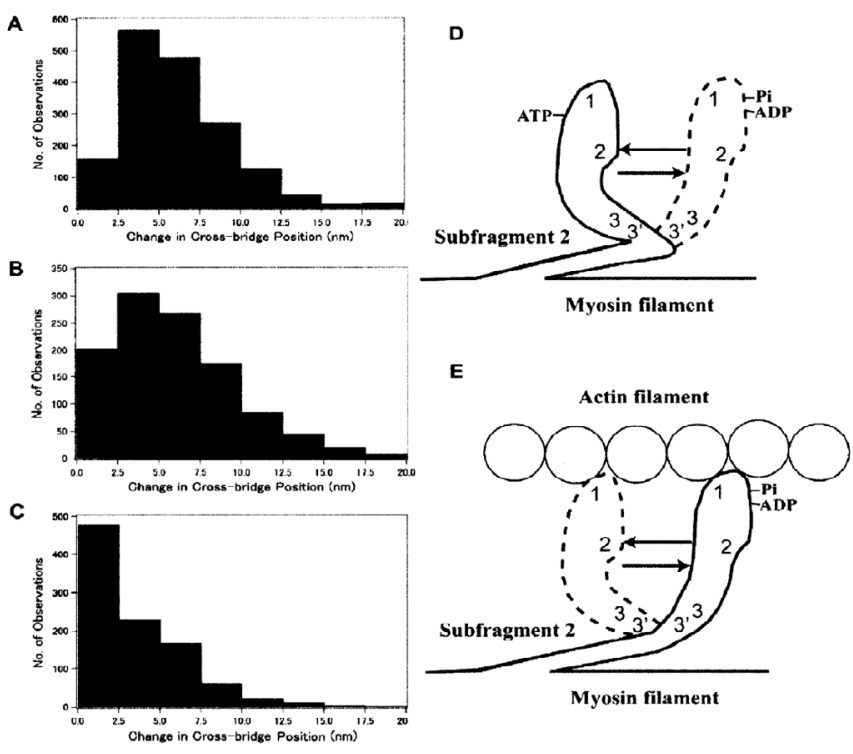

Figure 13: $(\mathrm{A}-\mathrm{C})$ Histograms showing the amplitude distribution of ATPinduced myosin head movement, position-marked with antibody $1(A)$, antibody $2(B)$, and antibody $3(C)$. (D and E) Diagrams illustrating myosin head lever arm mechanism in the absence of actin filament (D) and in the presence of actin filament $(\mathrm{E})$. Approximate attachment regions of antibodies 1,2 and 3 are indicated by numbers 1,2 and 3 and 3', respectively [26]

corresponding different particles are located, are shown in Figure 11. In each frame, pixel positions in the first (red), second (blue), and third (yellow) IP records can be taken to indicate sequential position changes of individual myosin heads. It can be seen that myosin heads first move in response to applied ATP, associated with reaction, $\mathrm{M}+\mathrm{ATP} \rightarrow$ $\mathrm{M} \cdot \mathrm{ATP} \rightarrow \mathrm{M} \cdot \mathrm{ADP} \cdot \mathrm{Pi}$, and after exhaustion of applied ATP return toward their initial position before ATP application, i.e. detachment of $\mathrm{Pi}$ and ADP from M, M.ADP.Pi $\rightarrow \mathrm{M}+\mathrm{ADP}+\mathrm{Pi}$. When the amplitude of ATP-induced myosin head movement is small $(\sim 5 \mathrm{~nm})$, myosin heads return almost exactly to its initial position, as indicated by complete overlap of pixel positions in the first and third records; yellow pixels are completely covered by red pixels (Figure $11 \mathrm{AB}$ ). If the amplitude of myosin head movement is large, the return of myosin heads to their initial position is incomplete (Figure $11 \mathrm{C}-\mathrm{H}$ ). These results indicate that ATP-induced myosin head movement is reversible; myosin heads in the state of M.ADP.Pi return toward their initial position after releasing $\mathrm{Pi}$ and ADP [24]. The myosin head movement during its return to the initial position may correspond to its power stroke in the presence of actin filament.

Novel features of myosin head movement as revealed by experiments with the EC: As described above, the experiments with the EC have revealed some novel features of myosin head movement. First of all, it has become clear that, even in the absence of actin filaments, myosin heads can perform cyclic movement without being guided by actin filaments. This implies that each myosin head (including S2 connecting it to myosin filament backbone) in itself has ability to perform ATP-dependent power and recovery strokes irrespective of whether actin filaments are present or absent. For this purpose, its stable equilibrium position may be essential.

In our EC experiments performed in the absence of actin filaments, the condition of myosin heads after application of ATP resembles the condition of myosin heads in relaxed muscle, in which myosin heads are not allowed to interact with actin filaments due to steric hindrance by tropomyosin [25]. In both conditions, myosin heads are in the state of M.ADP.Pi with a long average lifetime (>10s), and on detachment of ADP and Pi from M, myosin heads may perform movement similar to that of power stroke without interacting with actin filaments.

Amplitude of ATP-induced movement at various regions within a myosin head: Figure 12 is a ribbon diagram showing the structure of a myosin head, consisting of catalytic domain (CAD) containing actin-binding and ATPase sites, converter domain (COD), and lever arm domain (LD), connected to myosin filament backbone via S2. Mainly based on crystallographic studies on nucleotide-dependent structural changes of myosin heads detached from myosin filaments, it has been suggested that myosin head power stroke is produced by active rotation of $\mathrm{LD}$ around COD, while CAD remains rigid [15]. To ascertain this hypothesis, we have made experiments with the EC using three different antibodies (IgG) directed to three different regions within a myosin head. Antibody 1 is identical with that used in our previous experiments with the EC [4,24], and attaches to junctional peptide between 50 - and $20-\mathrm{kDa}$ segments of myosin heavy chain. Antibody 2 attaches around reactive lysine residue (Lys 83) in COD. Antibody 3 attaches to two peptides (Met 58-Ala 70 and Leu 106-Phe $120)$ in myosin regulatory light chain in LD. Regions of attachment of antibodies 1,2 and 3 are indicated by numbers 1,2 and 3 in Figure 12.

The results are summarized in Figure 13 together with their interpretation. Figure 13A-C are histograms of amplitude distribution of ATP-induced movement of myosin heads, position-marked with antibody 1, antibody 2 and antibody 3, respectively. The mean amplitude of ATP-induced movement is $6.14 \pm 0.09 \mathrm{~nm}$ (mean \pm SEM, $\mathrm{n}=1692)$ at the actin-binding site in CAD, $6.14 \pm 0.22(\mathrm{n}=1112)$ at the boundary between CAD and COD, and $3.55 \pm 0.11(n=981)$ [26]. These results indicate that the mean amplitude of ATP-induced movement does not differ significantly between the distal and proximal regions of myosin head CAD, but is much smaller in myosin head lever arm region. It follows from this that, during myosin head movement, myosin head CAD remains rigid, as has been expected from the lever arm mechanism of myosin head movement [15]. Thus, the EC experiments combined with the use of site-directed antibodies enables us to study 
regional difference in the amplitude of movement at various regions within a macromolecule such as myosin heads.

We think that cyclic conformational changes of myosin heads coupled with ATP hydrolysis may almost be the same in both absence (Figure 13D) and presence (Figure 13E) of actin filaments, since the ATP-induced myosin head movement are reversible (Figure 11), and the direction of movement is away from myosin filament bare region (Figure 10), being consistent with the scheme of cyclic actin-myosin interaction coupled with ATP hydrolysis (Figure 5). As already mentioned, our experiments with the EC have clearly shown that cyclic myosin head movement coupled with ATP hydrolysis is incorporated within myosin head structure, but is not guided by actin filaments to which myosin heads attach.

Recently, we have succeeded in recording myosin head power stroke in the presence of actin filaments, using the mixture of actin and myosin filaments [27]. The amplitude of power stroke in individual myosin heads is found to be $\sim 3 \mathrm{~nm}$ in the isometric condition in which gross myofilament sliding is inhibited. The amplitude of myosin head power stroke increases to $\sim 5 \mathrm{~nm}$ at low ionic strength, in accordance with physiological experiments that the amplitude of $\mathrm{Ca}^{2+}$-activated isometric force in skinned muscle fibers increases twofold at low ionic strength [28]. The experiments are currently in progress with a number of novel results, including regional difference in the amplitude of ATPinduced power stroke within individual myosin heads. This proves our EC system to be an extremely powerful tool in revealing novel features on the performance of myosin heads in rower myosins, which are difficult to investigate by experimental methods effective for studying processive movement of porter myosins.

\section{Future Prospects}

As described in this article, the EC enables us to study dynamic structural changes in living, hydrated biological macromolecules retaining their physiological function under a high magnification of electron microscope. In addition to silicon nitride sealing film, which can be commercially available, systems to record electron microscopic images of biological specimens have been developed for general use of investigators $[2,3]$. We hope that the EC will widely be used in the research field of life sciences to give answers to a number of mysteries concerning structure and function of biological macromolecules.

It should be noted, however, that the use of the EC has absolute limitation with respect to the critical incident electron dose to give damage to biological specimen. The damage may be partly due to direct damage to molecules constituting the specimen, and partly due to production of free radicals to impair physiological function of the specimen. The effect of free radicals may be reduced to some extent by use of anti-oxidant such as glutathione.

In our present experiments with the EC, more than $80 \%$ of the critical electron dose should be used to find and focus myofilaments under low magnifications of electron microscope. If a device is developed to find and focus suitable specimens rapidly (for example, by using a computer monitoring the shape of suitable specimens), then it would become possible to take a number of electron microscopic records on one and the same specimen; as the result, we may make animations visualizing time consequence of structural changes of the specimen related to its physiological function.

Also due to limitation of critical electron dose, we limit electron microscopic magnification to be $10,000 x$. In this experimental condition, the limit of accuracy of IP recording system is $\sim 0.6 \mathrm{~nm}$ as already mentioned. We think that this value cannot be improved appreciably, if larger electron microscopic magnification can be made to increase together with increased number of pixels in the recording system. Since the number of electrons entering to each pixel decreases with increasing magnification and also with increasing number of pixels, the special resolution may not be improved markedly. The problems concerning the use of the IP would, however, be very much improve by the use of recently developed slow CCD camera, in which background noise inherent to IP system is very much reduced $[29,30]$.

In addition to the use of slow CCD instead of IP, another way to improve special resolution of the EC system is to amplify minute structural changes in biological molecules by some means. We find an ingenious example of amplifying structural changes, in the work of Kinosita et al. [31]. They made a success in visualizing rotary motion of $\mathrm{F}_{0} \mathrm{~F}_{1}$ ATPase complex isolated from mitochondrial membrane under a light microscope by attaching fluorescently labeled actin filaments to its rotating region. This method may be applicable to other specimens playing important roles in cell function.

\section{Acknowledgement}

We wish to thank President Kazuo Ito, President Terukazu Etoh, and Presiden Yoshiyasu Harada of Japan Electron Optics Co. Ltd. for generously providing facilities to carry out our experimental work. The names of our coworkers are listed below with thanks; Drs. Tsuyoshi Akimoto, Kazuo Sutoh, Yuri Inayoshi, Tatsuhiro Okabe, Fumiaki Yumoto, Noboru Oishi, Suechika Suzuki, Yumiko Miyauchi and Seiryo Sugiura in the work published in 1997, 2008 and 2011.

\section{References}

1. Buttler EP, Hale KF (1981) Dynamic experiments in the electron microscope: Practical methods in electron microscopy. Vol 9, North Holland, Amsterdam.

2. de Jonge N, Peckys DB, Kremers GJ, Piston DW (2009) Electron microscopy of whole cells in liquid with nanometer resolution. Proc Natl Acad Sci U S A 106: $2159-2164$

3. Mirsaidov UM, Zheng H, Casana Y, Matsudaira P (2012) Imaging protein structure in water at $2.7 \mathrm{~nm}$ resolution by transmission electron microscopy. Biophys J 102: L15-17.

4. Sugi H, Akimoto T, Sutoh K, Chaen S, Oishi N, et al. (1997) Dynamic electron microscopy of ATP-induced myosin head movement in living muscle thick filaments. Proc Natl Acad Sci U S A 94: 4378-4382.

5. Fukami A, Fukushima K, Kohyama N (1991) Observation technique for we clay minerals using fine-sealed environmental cell equipment attached to highresolution electron microscope. Microstructure of fine-grained sediments from mud to shale. Frontiers in Sedimentary Geology 321-331.

6. Fukushima K (1988) Application of the gas environmental chamber for electron microscopy. PhD Thesis (Nagoya University, Nagoya).

7. Fukami A, Adachi K (1965) A new method of preparation of a self-perforated micro plastic grid and its application. J Electron Microsc (Tokyo) 14: 112-118.

8. Sugi H, Minoda H, Miyakawa T, Tanokura S, Chaen S, et al. (1912) The gas environmental chamber as a powerful tool to study structural changes of living muscle thick filaments coupled with ATP hydrolysis. Current basic and pathological approaches to the function of muscle cells and tissues-From molecules to humans. InTech, Rijeka, Croatia.

9. Huxley $\mathrm{H}$, Hanson $\mathrm{J}$ (1954) Changes in the cross-striations of muscle during contraction and stretch and their structural interpretation. Nature 173: 973-976.

10. Sugi H (1992) Molecular mechanism of actin-myosin interaction in muscle contraction. Muscle contraction and cell motility: Molecular and cellular aspects. Springer, Berlin and Heidelberg.

11. Huxley HE (2002) The mechanism of muscular contraction. Science 164:13561366, 1969. Clin Orthop Relat Res : S6-17.

12. Cooke R (1986) The mechanism of muscle contraction. CRC Crit Rev Biochem 21: 53-118.

13. Hibberd MG, Trentham DR (1986) Relationships between chemical and mechanical events during muscular contraction. Annu Rev Biophys Biophys Chem 15: 119-161. 
Citation: Sugi H, Miyakawa T, Tanokura S, Chaen S, Minoda H, et al. (2014) Visualization and Recording of Structural Changes in Hydrated, Living Muscle Myofilaments using the Gas Environmental Chamber. J Nanomed Nanotechnol S5:005. doi:10.4172/2157-7439.S5-005

Page 9 of 9

14. Huxley AF (1998) Muscle. Support for the lever arm. Nature 396: 317-318.

15. Geeves MA, Holmes KC (1999) Structural mechanism of muscle contraction. Annu Rev Biochem 68: 687-728.

16. Lymn RW, Taylor EW (1971) Mechanism of adenosine triphosphate hydrolysis by actomyosin. Biochemistry 10: 4617-4624.

17. Bagshaw CR (1993) Muscle contraction. Chapman \& Hall, London.

18. Sugi $H$ (2006) Remaining mysteries about the sliding filament mechanism in muscle contraction. Recent Res Devel Biophys 5: 167-186.

19. Yildiz A, Forkey JN, McKinney SA, Ha T, Goldman YE, et al. (2003) Myosin $\checkmark$ walks hand-over-hand: single fluorophore imaging with 1.5-nm localization. Science 300: 2061-2065

20. Mehta AD, Rock RS, Rief M, Spudich JA, Mooseker MS, et al. (1999) Myosin-V is a processive actin-based motor. Nature 400: 590-593.

21. Kodera N, Yamamoto D, Ishikawa R, Ando T (2010) Video imaging of walking myosin $\mathrm{V}$ by high-speed atomic force microscopy. Nature 468: 72-76.

22. Suda H, Ishikawa A, Fukami A (1992) Evaluation of the critical electron dose on the contractile activity of hydrated muscle fibers in the film-sealed environmental cell. J Electron Microsc (Tokyo) 41: 223-229.

23. Sutoh K, Tokunaga M, Wakabayashi T (1989) Electron microscopic mappings of myosin head with site-directed antibodies. J Mol Biol 206: 357-363.

24. Sugi $H$, Minoda $H$, Inayoshi $Y$, Yumoto $F$, Miyakawa $T$, et al. (2008) Direct demonstration of the cross-bridge recovery stroke in muscle thick filaments in aqueous solution by using the hydration chamber. Proc Natl Acad Sci U S A 105: $17396-17401$.
25. Ebashi S, Endo M (1968) Calcium ion and muscle contraction. Prog Biophys Mol Biol 18: 123-183.

26. Minoda H, Okabe T, Inayoshi Y, Miyakawa T, Miyauchi Y, et al. (2011) Electron microscopic evidence for the myosin head lever arm mechanism in hydrated myosin filaments using the gas environmental chamber. Biochem Biophys Res Commun 405: 651-656.

27. Sugi $H(2013)$ Visualization and measurement of the power stroke in individual myosin heads coupled with ATP hydrolysis using the gas environmental chamber. J PhysiolSci 103 Suppl : S35.

28. Sugi H, Abe T, Kobayashi T, Chaen S, Ohnuki Y, et al. (2013) Enhancement of force generated by individual myosin heads in skinned rabbit psoas muscle fibers at low ionic strength. PLoS One 8: e63658.

29. Yasunaga T, Wakabayashi T (2008) Evaluation of a $2 k$ CCD camera with an epitaxially grown Csl scintillator for recording energy-filtered electron cryomicrographs. J Electron Microsc (Tokyo) 57: 101-112.

30. Murakami K, Yasunaga T, Noguchi TQ, Gomibuchi Y, Ngo KX, et al. (2010) Structural basis for actin assembly, activation of ATP hydrolysis, and delayed phosphate release. Cell 143: 275-287.

31. Kinosita K Jr, Ali MY, Adachi K, Shiroguchi K, Itoh H (2005) How two-foot molecular motors may walk. Adv Exp Med Biol 565: 205-218.
This article was originally published in a special issue, Nanotechnology: Challenges \& Perspectives in Medicine handled by Editor(s). Dr. Malavosklish Bikram, University of Houston, USA 